\title{
Report of four novel variants in ASNS causing asparagine synthetase deficiency and review of literature
}

\author{
Chelna Galada $^{1}$, Malavika Hebbar ${ }^{1}$, Leslie Lewis ${ }^{2}$, Santosh Soans $^{3}$, Rajagopal Kadavigere ${ }^{4}$, Anshika Srivastava ${ }^{5}$, \\ Stephanie Bielas ${ }^{5}$, Katta M. Girisha ${ }^{1}$, and Anju Shukla ${ }^{1}$ (D) \\ Departments of ${ }^{1}$ Medical Genetics, ${ }^{2}$ Pediatrics, ${ }^{4}$ Radiodiagnosis and Imaging, Kasturba Medical College, Manipal Academy of Higher \\ Education, Manipal, ${ }^{3}$ Department of Paediatrics, A.J. Institute of Medical Sciences and Research Centre, Mangalore, India and \\ ${ }^{5}$ Department of Human Genetics, University of Michigan, Ann Arbor, Michigan, USA
}

Asparagine synthetase deficiency (ASNSD, MIM 615574) is a recently delineated rare neurometabolic disorder caused by mutations in ASNS (MIM 108370) (Ruzzo et al. 2013). It is characterized by congenital and/or postnatal progressive microcephaly, global developmental delay, seizures, growth retardation, cerebral atrophy, and simplified gyral pattern. We hereby describe four novel variations ascertained by exome sequencing in three individuals from three unrelated families (Fig. S1, Supporting information). We summarize the genotype and phenotype of 22 individuals from 14 families with 16 mutations in ASNS (Ruzzo et al. 2013; Alfadhel et al. 2015; Ben-Salem et al. 2015; Palmer et al. 2015; Gataullina et al. 2016; Seidahmed et al. 2016; Gupta et al. 2017; Sun et al. 2017; Yamamoto et al. 2017). Informed consents from the families were obtained for the study. The study was approved by the Institutional Ethics Committee. Detailed clinical information, exome sequencing methodology, and analysis are provided in Appendix S1.

All three individuals showed congenital microcephaly, progressive postnatal microcephaly, and spasticity, whereas seizures were noted only in patients 1 and 3 (Table S1). Magnetic resonance imaging of brain revealed simplified gyral pattern, cerebral atrophy, and pontocerebellar hypoplasia in all patients (Fig. 1). Whole exome sequencing (WES) analysis in patient 1 revealed a novel homozygous missense variant, c.1211G >A [p.(Arg404His)], in exon 10 of ASNS gene. Analysis of patient 2 disclosed novel compound heterozygous variants, c.224A $>\mathrm{G}$ [p.(Asn75Ser)] in exon 3 and c.413A $>$ C [p.(Asp138Ala)] in exon 4 of $A S N S$ gene. In patient 3, a novel homozygous variant, c.1649G>A [p. (Arg550His)], was observed. These variants are not present in either homozygous or heterozygous states in databases of normal population. These variants are also not observed in our in-house

Correspondence: Anju Shukla, MD (Pediatrics), DM (Medical Genetics) Department of Medical Genetics, Kasturba Medical College, Manipal Academy of Higher Education, Manipal 576104, Karnataka, India. Email: anju.shukla@manipal.edu

Received September 28, 2017; revised and accepted January 30, 2018. database of 357 families. These variants occur at a highly conserved region and predicted as damaging to ASNS protein function. Further analysis by in silico or any other functional assay could not be performed for these variants.

A review of 25 individuals including our patients revealed significant global developmental delay $(22 / 23)$ in most individuals. Congenital microcephaly and simplified gyral pattern on brain imaging was evident in a significant proportion (19/25) of individuals. Other frequent brain imaging findings were pontocerebellar hypoplasia/reduced pons size (16/25) and progressive cerebral atrophy seen in all the individuals. Spastic quadraparesis (19/24) and seizures (18/25) are the other common findings in individuals with ASNSD (Table S1). High degree of inbreeding observed in the local population is the possible cause of homozygous variants in non-consanguineous families in our study.

We herein emphasize the utility of whole exome sequencing as an important tool in delineating the genetic etiology in individuals with rare and clinically heterogeneous phenotypes. We also add four novel mutations, thus expanding the ASNS mutation spectrum. The presence of variable degree of congenital microcephaly underscores that this condition should be considered among the various genetic disorders presenting with primary microcephaly.

\section{ACKNOWLEDGMENTS}

We thank the families who consented for participation in this study. This study was supported by National Institutes of Health, which funded the project titled "Genetic Diagnosis of Heritable Neurodevelopmental Disorders in India: Investigating the Use of WholeExome Sequencing and Genetic Counseling to Address the High Burden of Neurodevelopmental Disorders" (1R21NS094047-01).

\section{DISCLOSURES}

None. 
Patient 1

(A)
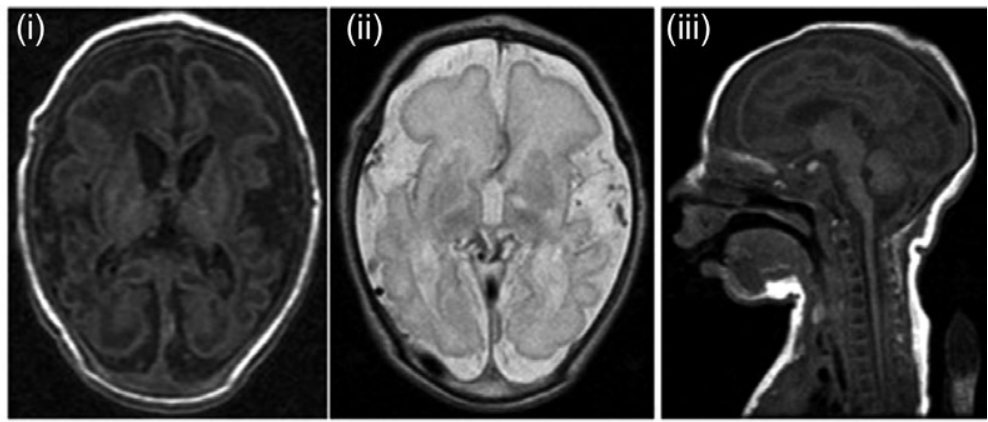

Fig. 1 (A) Brain magnetic resonance imaging (MRI) of patient 1 taken on day 2 of life. Axial T1- (i), axial T2- (ii), and sagittal T1 (iii)weighted images show microcephaly with overlapping of sutures, simplified gyral pattern, delayed myelination, reduced volume of the pons, and mild cerebral atrophy. (B) Brain MRI images of patient 2 taken at 1 month of age. Axial T1- (i), axial T2- (ii) and sagittal T2 (iii)weighted images show microcephaly with overlapping sutures, mild delayed myelination, reduced size of the pons, and cerebral atrophy. (C) Brain MRI images of patient 3 on day 2 of life. Axial T1- (i), coronal T2- (ii) and sagittal T1 (iii)-weighted images show microcephaly with overlapping of sutures, simplified gyral pattern, reduced volume of the pons, and cerebral atrophy.
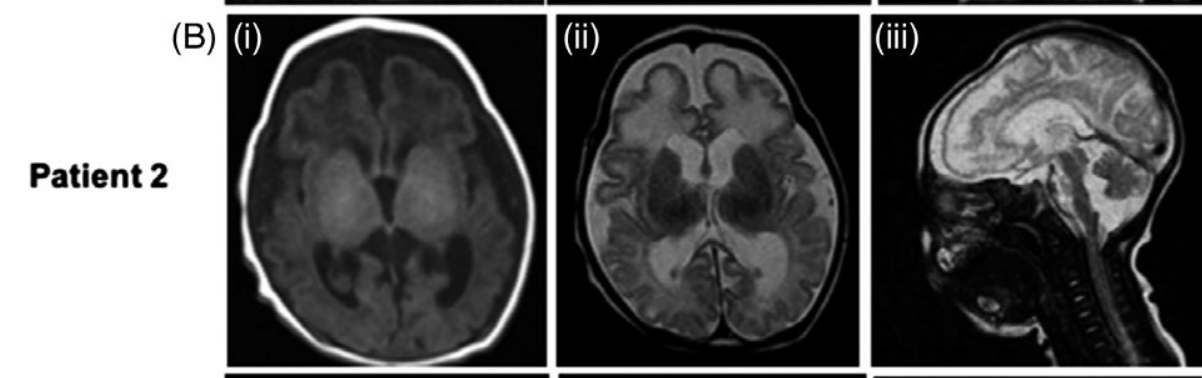

(C)
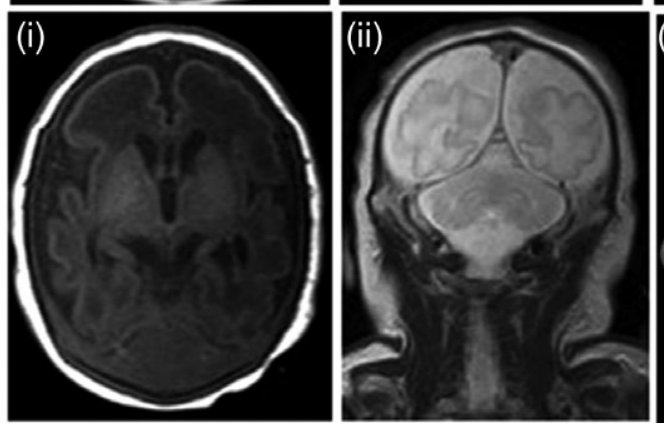

\section{REFERENCES}

Alfadhel M, Alrifai MT, Trujillano D et al. 2015. Asparagine synthetase deficiency: new inborn errors of metabolism. JIMD Rep 22:11-16.

Ben-Salem S, Gleeson JG, Al-Shamsi AM et al. 2015. Asparagine synthetase deficiency detected by whole exome sequencing causes congenital microcephaly, epileptic encephalopathy and psychomotor delay. Metab Brain Dis 30:687-694.

Gataullina S, Lauer-Zillhardt J, Kaminska A et al. 2016. Epileptic phenotype of two siblings with asparagine synthesis deficiency mimics neonatal pyridoxine-dependent epilepsy. Neuropediatrics 47:399-403.

Gupta N, Tewari VV, Kumar M et al. 2017. Asparagine synthetase deficiency-report of a novel mutation and review of literature. Metab Brain Dis 32:1889-1900.

Palmer EE, Hayner J, Sachdev R et al. 2015. Asparagine synthetase deficiency causes reduced proliferation of cells under conditions of limited asparagine. Mol Genet Metab 116:178-186.

Ruzzo EK, Capo-Chichi JM, Ben-Zeev B et al. 2013. Deficiency of asparagine synthetase causes congenital microcephaly and a progressive form of encephalopathy. Neuron 80:429-441.

Seidahmed MZ, Salih MA, Abdulbasit OB et al. 2016. Hyperekplexia, microcephaly and simplified gyral pattern caused by novel ASNS mutations, case report. BMC Neurol 16:105.
Sun J, McGillivray AJ, Pinner J et al. 2017. Diaphragmatic eventration in sisters with asparagine synthetase deficiency: a novel homozygous ASNS mutation and expanded phenotype. JIMD Rep 34:1-9.

Yamamoto T, Endo W, Ohnishi $\mathrm{H}$ et al. 2017. The first report of Japanese patients with asparagine synthetase deficiency. Brain Dev 39:236-242.

\section{SUPPORTING INFORMATION}

Additional supporting information may be found online in the supporting information tab for this article.

Appendix S1. Details of clinical data and exome sequencing. Fig. S1. Family pedigrees and mutations in the three families with asparagine synthetase deficiency (A-C). Patient 2 is a compound heterozygous for $A S N S$ variation where father is heterozygous for c. $413 \mathrm{~A}>\mathrm{C}$ and mother is heterozygous for c.224A $>\mathrm{G}$ variant in ASNS (B).

Table S1. Phenotype and genotype information of individuals in the present study and previously reported 22 individuals. 